\title{
ON THE UNIVALENCE OF FUNCTIONS WHOSE DERIVATIVE HAS A POSITIVE REAL PART
}

\author{
F. HERZOG AND G. PIRANIAN
}

1. Introduction and summary. If $D$ is a domain in the finite complex plane and $f(z)$ is holomorphic in $D$, the nonvanishing of $f^{\prime}(z)$ in $D$ is a necessary condition for the univalence of $f(z)$ in $D$. That the condition is not sufficient follows from the fact that the function $e^{z}$ is not schlicht in the domain $|z|<R$, when $R>\pi$. On the other hand, it was shown by K. Noshiro [1, Theorem 12 , p. 151 $]^{1}$ and by S. E. Warschawski [2, Lemma 1, p. 312] (the Noshiro-Warschawski Theorem being a generalization of a previous result by J. Wolff [3]) that every convex domain $D$ has the property that

(U) $f(z)$ is schlicht in $D$ whenever $\Re f^{\prime}(z)>0$ throughout $D$.

There exist nonconvex domains that have property (U)-any domain obtained by deleting a finite point-set from a convex domain will do. On the other hand, if $\pi / 2<\beta \leqq \pi$, the domain defined by the inequality $-\beta<\arg z<\beta$ does not have the property (U), as is seen by examining the function $f(z) \equiv z^{1+\pi / 2 \beta}$ (with $f(1)=1$ ). We shall show that if a domain has the property (U) it does not fall far short of being convex.

In order to describe our results in more convenient language, we introduce the following definitions: Let $D$ be a domain and $H$ the interior of its convex hull; the set of deficiency of $D$ with respect to convexity (more briefly: the set of deficiency of $D$ ) shall be the complement of $D$ relative to $H$. It is obvious that the set of deficiency of $D$ is closed relative to $H$ and that it is empty if and only if $D$ is convex; also that the set of deficiency of a domain is the minimal set whose union with the domain is convex. A domain shall be said to be almost convex provided every pair of open circles in $D$ can be connected by a line segment lying entirely in $D$. The following constitutes our main result. (See Theorems 1 and 2.)

In order that a domain $D$ have property (U), it is necessary that the set of deficiency of $D$ be totally disconnected and sufficient that $D$ be almost convex.

This result does not characterize the domains that have property (U); for there exist domains that fail to be almost convex although their sets of deficiency are totally disconnected. (See \$4, Case (iii).) But it does characterize the domains with property (U) among the

Received by the editors August 23, 1950.

1 Numbers in brackets refer to the bibliography at the end of the paper. 
domains of finite connectivity:

$A$ domain of finite connectivity has the property (U) if and only if its set of deficiency is finite or empty.

2. Domains whose sets of deficiency are not totally disconnected. A set is not totally disconnected provided it contains a nondegenerate continuum, that is, a connected closed set consisting of at least two points.

Theorem 1. A domain whose set of deficiency is not totally disconnected does not have property (U).

It is to be shown that, if the set of deficiency $S$ of the domain $D$ contains a nondegenerate continuum, there exists a function $f(z)$ not schlicht in $D$, but with the real part of its derivative positive throughout $D$. The proof is by construction. We deal separately with two cases:

(I) $S$ has interior points;

(II) each point of $S$ is a boundary point of $D$.

In dealing with Case (I), we need the following geometrical proposition.

Lemma. If the set of deficiency of a domain $D$ has interior points, there exists a circular domain, exterior to $D$, whose boundary meets the boundary of $D$ in at least two points.

In proving this lemma, the coordinates may be chosen in such a way that the origin is an interior point of $S$, the set of deficiency of $D$. The transformation $z^{*}=1 / z$ then maps the domain $D$ in the $z$-plane into a bounded domain $D^{*}$ in the $z^{*}$-plane. Let $\Gamma^{*}$ be the open circular disc of least radius that contains $D^{*}$, and let $\Gamma$ be the image in the $z$-plane of $\Gamma^{*}$; then the boundary of $\Gamma$ meets the boundary of $D$ in at least two points. Since the point at infinity in the $z^{*}$-plane is exterior to $\Gamma^{*}$, the origin in the $z$-plane is exterior to $\Gamma$. This implies that $\Gamma$ is the outside of a circle $K$; for otherwise $\Gamma$ would be a half-plane or a circular disc, and since $\Gamma$ contains $D$, the origin would be exterior to the convex hull of $D$, contrary to the hypothesis. It follows that the inside of $K$ has the required properties.

Suppose, then, that $S$ has interior points and that $C$ is a circle passing through at least two boundary points of $D$ and containing no points of $D$ in its inside. Again two cases arise:

(Ia) there exist points on $C$ that are not boundary points of $D$;

(Ib) all points on $C$ are boundary points of $D$.

The particular choice of coordinates used in proving the lemma will now be discarded, and at each stage we shall use whatever coordinate 
system is most suitable to our purpose.

Case (Ia). Let $C$ be identical with the unit circle; let $0<\alpha<\pi$, and let $e^{i \alpha}$ and $e^{-i \alpha}$ be boundary points of $D$, while the open arc on $C$ lying between them and passing through the point $z=1$ is free of boundry points of $D$.

Let $L_{0}$ denote the lemniscate $r^{2}=\cos 2 \theta$, where $r$ and $\theta$ are polar coordinates in the z-plane; then the distance between $D$ and the right loop of $L_{0}$ is positive. Now for every pair of points $z_{1}$ and $z_{2}$ that lie near enough to $e^{i \alpha}$ and $e^{-i \alpha}$, respectively, there exists a unique lemniscate $L\left(z_{1}, z_{2}\right)$ with the following properties:

(i) the center and vertices of $L\left(z_{1}, z_{2}\right)$ lie on the perpendicular bisector of the line segment joining $z_{1}$ and $z_{2}$;

(ii) the left loop of $L\left(z_{1}, z_{2}\right)$ is tangent to the unit circle $C$ from the inside of $C$;

(iii) the circle which is concentric with $L\left(z_{1}, z_{2}\right)$ and passes through its vertices also passes through the points $z_{1}$ and $z_{2}$.

As $z_{1} \rightarrow e^{i \alpha}$ and $z_{2} \rightarrow e^{-i \alpha}, L\left(z_{1}, z_{2}\right)$ approaches $L_{0}$ in the obvious sense of the word; it is therefore possible to choose $z_{1}$ and $z_{2}$ in $D\left(z_{1} \neq z_{2}\right)$ in such a way that no point of $D$ lies inside or on $L\left(z_{1}, z_{2}\right)$.

Having chosen such a pair of points, we use a transformation $\zeta=a z$ $+b$ to map $L\left(z_{1}, z_{2}\right)$ onto the lemniscate $\Lambda_{0}$ whose equation in the polar coordinates of the $\zeta$-plane is $\rho^{2}=\cos 2 \phi$. By $\zeta_{1}, \zeta_{2}$, and $\Delta$, respectively, we denote the images of $z_{1}, z_{2}$, and $D$ under this transformation. The points $\zeta_{1}$ and $\zeta_{2}$ are then distinct points in $\Delta$, conjugate to one another and of unit modulus; and the lemniscate $\Lambda_{0}$ contains no points of $\Delta$. If $g(\zeta) \equiv \zeta+\zeta^{-1}$, then $g\left(\zeta_{1}\right)=g\left(\zeta_{2}\right)$ and $\Re g^{\prime}(\zeta)=1$ $-\rho^{-2} \cos 2 \phi>0$ for all points $\zeta$ outside of $\Lambda_{0}$, in particular, for all points of $\Delta$. It follows that the function

$$
f(z) \equiv \bar{a} g(\zeta)=\bar{a}\left\lfloor(a z+b)+(a z+b)^{-1}\right\rfloor
$$

has the required properties.

Case (Ib). Again let $C$ be the unit circle. We use the function

$$
f(z) \equiv \eta z-\left(z^{2}-a^{2}\right)^{-1 / 2}=\eta z-z^{-1}\left(1-a^{2} / z^{2}\right)^{-1 / 2},
$$

where $\eta$ and $a$ are real constants whose values are to be determined below, subject to the conditions $\eta>1,0<a<1$; and where $\left(1-a^{2} / z^{2}\right)^{-1 / 2}$ has the value one at $z=\infty$. In the domain $|z|>a, f(z)$ is holomorphic and odd.

From(1) it follows that

$$
f^{\prime}(z)=\eta+z^{-2}+\frac{3}{2} a^{2} z^{-4}+\sum_{n=2}^{\infty} c_{n} a^{2 n} z^{-2 n-2},
$$


where $c_{n}=(3 / 2)(5 / 4) \cdots[(2 n+1) / 2 n]$. The function $f^{\prime}(z)$ is holomorphic for $|z| \geqq 1$, including $z=\infty$; if we can choose the constant $a$ so that $\Re f^{\prime}(z)>0$ for $|z|=1$, it will follow that $\Re f^{\prime}(z)>0$ for $|z|>1$, hence also for $z \in D$. With the notation $z=e^{i \theta}(0 \leqq \theta<2 \pi)$, we obtain the relation

$$
\begin{aligned}
\Re f^{\prime}\left(e^{i \theta}\right) & =\eta+\cos 2 \theta+\frac{3}{2} a^{2} \cos 4 \theta+\sum_{n=2}^{\infty} c_{n} a^{2 n} \cos (2 n+2) \theta \\
& >\phi(t)-\sum_{n=2}^{\infty} c_{n} a^{2 n},
\end{aligned}
$$

where $t=\cos 2 \theta$ and $\phi(t) \equiv 1-3 a^{2} / 2+t+3 a^{2} t^{2}$. If $a$ is sufficiently small, $\phi^{\prime}(t)>0$ for $-1 \leqq t \leqq 1$ and, therefore, $\phi(t) \geqq \phi(-1)=3 a^{2} / 2$. It follows that, for $0 \leqq \theta<2 \pi$,

$$
\Re f^{\prime}\left(e^{i \theta}\right)>\frac{3}{2} a^{2}-\sum_{n=2}^{\infty} c_{n} a^{2 n},
$$

and the last member is clearly positive for sufficiently small values of a.

Suppose, then, that the constant $a$ has been chosen small enough so that $\Re f^{\prime}(z)>0$ throughout $D$ for every choice of the constant $\eta(\eta>1)$. Let the real constant $\beta$ be chosen greater than one and sufficiently near to one so that

$$
\left(\gamma^{2}-a^{2}\right)^{-1 / 2}>\gamma \quad \text { for } 1<\gamma<\beta .
$$

Since every point of $C$ is a boundary point of $D$, we can find two points in $D$, lying on opposite rays from the origin and as near to $C$ as we wish; with appropriate choice of the coordinate system, these points lie on the real axis and have coordinates $\gamma_{1}$ and $-\gamma_{2}$, with $1<\gamma_{j}<\beta$. Then

$$
\begin{aligned}
f\left(\gamma_{1}\right) & =\eta \gamma_{1}-\left(\gamma_{1}^{2}-a^{2}\right)^{-1 / 2}, \\
f\left(-\gamma_{2}\right) & =-f\left(\gamma_{2}\right)=-\eta \gamma_{2}+\left(\gamma_{2}^{2}-a^{2}\right)^{-1 / 2},
\end{aligned}
$$

and therefore $f\left(\gamma_{1}\right)=f\left(-\gamma_{2}\right)$ provided we choose

$$
\eta=\frac{\left(\gamma_{1}^{2}-a^{2}\right)^{-1 / 2}+\left(\gamma_{2}^{2}-a^{2}\right)^{-1 / 2}}{\gamma_{1}+\gamma_{2}}
$$

By virtue of (2), this choice gives $\eta>1$, and the constants $a$ and $\eta$ have been chosen so that $f(z)$ has the required properties.

Case (II). Since $S$ contains a nondegenerate continuum, it contains 
a bounded continuum $K$; and we may assume that the coordinate system has been chosen in such a way that $K$ contains the points $z=1$ and $z=-1$. The complement $\tilde{K}$ of $K$, relative to the extended plane, is then a simply-connected domain containing $D$; and the function $\phi(z) \equiv z-\left(z^{2}-1\right)^{1 / 2}$ (with $\left.\phi(\infty)=0\right)$ is holomorphic and bounded in $\tilde{K}$. While the function $\phi(z)$ is not the derivative of a singlevalued function in $\tilde{K}$, its square does have this property and, therefore, we choose

$$
\begin{aligned}
f(z) & \equiv z+\omega \int[\phi(z)]^{2} d z \\
& =z+\frac{\omega}{3}\left[2 z^{3}-3 z-2\left(z^{2}-1\right)^{3 / 2}\right]
\end{aligned}
$$

where $\omega$ is a complex number yet to be determined. Since $f^{\prime}(z)=1$ $+\omega[\phi(z)]^{2}, f(z)$ has a positive real part throughout $\tilde{K}$ (and therefore throughout $D$ ) provided $|\omega|$ is less than a certain positive constant $\delta$.

Since each point of $K$ is a boundary point of $D$, there exists a point $c$ on $K\left(c^{2} \neq 1\right)$ which can be approached by sequences $\left\{z_{n}^{\prime}\right\}$ and $\left\{z_{n}^{\prime \prime}\right\}$ in $D$ in such a way that the two expressions $\left(z_{n}^{\prime 2}-1\right)^{3 / 2}$ and $\left(z_{n}^{\prime \prime 2}-1\right)^{3 / 2}$ approach the two distinct values of the symbol $\left(c^{2}-1\right)^{3 / 2}$. Therefore, as $n$ becomes large, that value of the constant $\omega$ for which the relation $f\left(z_{n}^{\prime}\right)=f\left(z_{n}^{\prime \prime}\right)$ holds approaches zero. If the index $n$ is large enough $\omega$ can be chosen subject to the condition $|\omega|<\delta$ and in such a way that $f\left(z_{n}^{\prime}\right)=f\left(z_{n}^{\prime \prime}\right)$. This completes the proof of Theorem 1 .

3. Almost convex domains. In this section we establish the second part of our main result and discuss a certain unsolved problem connected with it.

TheOREM 2. A domain which is almost convex has property (U).

It should be observed that Theorem 2 contains the NoshiroWarschawski Theorem (see \$1). To prove the theorem, suppose first that $z_{1}$ and $z_{2}$ are two distinct points of the domain $D$ such that $D$ contains the line segment joining the two points, and let $z_{2}=z_{1}+r e^{i \alpha}$ $(r>0)$. Then

$$
f\left(z_{2}\right)-f\left(z_{1}\right)=\int_{z_{1}}^{z_{2}} f^{\prime}(z) d z=e^{i \alpha} \int_{0}^{r} f^{\prime}\left(z_{1}+t e^{i \alpha}\right) d t .
$$

The real part of the last integral is positive and, therefore, $f\left(z_{2}\right)$ $\neq f\left(z_{1}\right)$.

If $z_{1}$ and $z_{2}$ are two points of $D$ that cannot be joined by a straight 
line segment in $D$, let $R$ be such that the circular discs $\left|z-z_{1}\right| \leqq R$ and $\left|z-z_{2}\right| \leqq R$ lie in $D$; and let $M$ and $\mu$ be positive constants such that the relations $\left|f^{\prime}(z)\right| \leqq M$ and $\Re f^{\prime}(z) \geqq \mu$ hold throughout these two discs. Let $\epsilon$ be a constant subject to the condition $0<\epsilon<R$ and to a further condition specified below; and let $\zeta_{1}$ and $\zeta_{2}$ be two points such that $\left|\zeta_{1}-z_{1}\right|<\epsilon$ and $\left|\zeta_{2}-z_{2}\right|<\epsilon$ and such that $D$ contains the line segment joining them. With the notation $\zeta_{2}=\zeta_{1}+\rho e^{i \beta}(\rho>0)$ we obtain, as above, the relation

$$
f\left(z_{2}\right)-f\left(z_{1}\right)=\int_{z_{1}}^{\zeta_{1}} f^{\prime}(z) d z+e^{i \beta} \int_{0}^{p} f^{\prime}\left(\zeta_{1}+t e^{i \beta}\right) d t+\int_{\zeta_{2}}^{z_{2}} f^{\prime}(z) d z .
$$

The first and the last integrals have moduli less than $M \epsilon$ each. The real part of the second integral is greater than $2(R-\epsilon) \mu$, hence the same is true of its modulus. If $\epsilon$ is chosen less than $R \mu /(\mu+M)$, it follows that $f\left(z_{2}\right) \neq f\left(z_{1}\right)$, and the theorem is proved.

We do not know of any function $f(z)$ whose derivative has a positive real part throughout some almost convex domain $D$, and which cannot be defined to be holomorphic in the interior of the convex hull of $D$. In other words, we do not know whether or not there exists a generalization of Riemann's Theorem on removable singularities which would permit Theorem 2 to be proved as an immediate consequence of the Noshiro-Warschawski Theorem. But we shall prove the required generalization of Riemann's Theorem for a large class of almost convex domains. In order to state our result more concisely, we make the following definition. A point-set $E$ in the plane has perimeter zero provided, for every positive number $\epsilon$, there exists a set of disjoint rectifiable closed Jordan curves $J_{k}$ such that each point of $E$ is enclosed by one of the curves $J_{k}$ and such that the sum of the lengths of the curves $J_{k}$ is less than $\epsilon$.

THEOREM 3. If $\psi(z)$ is holomorphic and bounded in a domain $D$ whose set of deficiency has perimeter zero, then $\psi(z)$ can be defined so as to be holomorphic in the interior of the convex hull of $D$.

Let $D$ be a domain and $S$ its set of deficiency, of perimeter zero. Let $s$ be an arbitrary point of $S$. It follows from the definition above that $s$ can be enclosed by a rectifiable closed Jordan curve $\Gamma$, lying entirely in $D$. Let $T$ be the part of $S$ that lies inside $\Gamma$. We shall show that $\psi(z)$ can be defined holomorphically on $T$, hence in particular at the point $s$.

If $\epsilon$ is small enough the corresponding curves $J_{k}$ which enclose $T$ lie entirely inside $\Gamma$. Also, by the Heine-Borel Theorem, it may be assumed that the set $\left\{J_{k}\right\}$ consists of finitely many curves. Any 
point $z$ inside $\Gamma$, at a distance $\delta$ greater than $\epsilon / 2$ from $T$, lies outside of each of the curves $J_{k}$; and, for any such point $z$, Cauchy's Integral Theorem gives the relation

$$
2 \pi i \psi(z)=\int_{\Gamma} \frac{\psi(\zeta) d \zeta}{\zeta-z}-\sum_{k} \int_{J_{k}} \frac{\psi(\zeta) d \zeta}{\zeta-z},
$$

where all integrals are to be taken in the counter-clockwise direction. Since the sum with respect to $k$ has modulus less than $M \epsilon /(\delta-\epsilon / 2)$, where $M$ is an upper bound for $|\psi(z)|$ in $D$, and the first integral is independent of $\epsilon$, the sum with respect to $k$ must be zero, and the remaining integral defines a function which is holomorphic inside $\Gamma$.

TheOREM 4. If the set of deficiency of the domain $D$ has perimeter zero and if $f(z)$ has a derivative whose real part is positive throughout $D$, then $f(z)$ can be defined so as to be holomorphic in the interior of the convex hull of $D$.

Under the hypotheses of the theorem, the function $\psi(z)$ $\equiv\left[1-f^{\prime}(z)\right] /\left[1+f^{\prime}(z)\right]$ has modulus less than one throughout $D$. By Theorem 3, the function $\psi(z)$ and therefore the function $f^{\prime}(z)$ can be defined so as to be holomorphic in $H$, the interior of the convex hull of $D$. Since $H$ is simply-connected, $f(z)$ can be extended so as to be holomorphic in $H$, and the theorem is proved.

TheOREM 5. If $D$ is a domain whose set of deficiency has perimeter zero, $D$ is almost convex.

Let $z_{1}$ and $z_{2}$ be any two points in $D$ and let $L$ be a line perpendicular to the line segment joining $z_{1}$ and $z_{2}$. Since the set of deficiency of $D$ has perimeter zero, its orthogonal projection upon $L$ must be a set of measure zero; hence the complement of the projection is everywhere dense on $L$. It follows that $D$ contains line segments perpendicular to $L$ and passing arbitrarily near $z_{1}$ and $z_{2}$. This proves the theorem.

The converse of Theorem 5 does not hold. For if the set of deficiency of $D$ is a totally disconnected linear set of positive measure, it does not have perimeter zero although $D$ is almost convex. A more interesting counter-example to the converse of Theorem 5 is described in the next section. (See $\$ 4$, Case (ii).)

4. Concerning certain Cantor sets. Let $b$ be a positive constant less than $1 / 2$ and let $S_{0}$ denote the unit square $0 \leqq x \leqq 1,0 \leqq y \leqq 1$. Let $S_{1}$ be the union of the four closed squares whose sides have length $b$ and which lie in the corners of $S_{0}$. When $S_{n-1}$ has been defined let $S_{n}$ be the union of the $4^{n}$ closed squares whose sides have length $b^{n}$ 
and which lie in the corners of the $4^{n-1}$ squares of $S_{n-1}$. Let $S$ be the intersection $S_{0} \cdot S_{1} \cdot S_{2} \cdots$, and let $D$ be the complement of $S$. Then

(i) if $0<b<1 / 4, S$ is of perimeter zero, hence $D$ is almost convex (by Theorem 5 );

(ii) if $b=1 / 4, D$ is almost convex, but $S$ is not of perimeter zero;

(iii) if $1 / 4<b<1 / 2, D$ is not almost convex and, therefore, $S$ is not of perimeter zero (by Theorem 5 ).

Case (i). It is possible to enclose $S_{n}$ by $4^{n}$ squares whose sides have length $\beta^{n}$, where $b<\beta<1 / 4$. The combined length of the perimeters of these squares is $4(4 \beta)^{n}$ and, therefore, $S$ is of perimeter zero.

Case (ii). We show first that $D$ is almost convex. The direction of any line that passes through the lower left-hand vertices of two squares of a set $S_{n}$ shall be called a preferred direction. If $\Lambda$ is a line perpendicular to a preferred direction, the orthogonal projection $S^{\prime}$ of $S$ upon $\Lambda$ shall be shown to have measure zero. Since the projection $S^{\prime}$ is the intersection of the projections $S_{0}^{\prime}, S_{1}^{\prime}, S_{2}^{\prime}, \cdots$, it will be sufficient to show that the measure of $S_{n}^{\prime}$ tends to zero for a subsequence of the sequence of integers $n$. For some index $p$, the projections upon $\Lambda$ of at least two squares of $S_{p}$ coincide and, therefore, $m S_{p}^{\prime} \leqq\left[\left(4^{p}-1\right) / 4^{p}\right] \cdot\left(m S_{0}^{\prime}\right)$. Similarly, $m S_{k p}^{\prime} \leqq\left[\left(4^{p}-1\right) / 4^{p}\right]^{k}\left(m S_{0}^{\prime}\right)$. It follows that $m S_{k p}^{\prime} \rightarrow 0$ as $k \rightarrow \infty$.

Let $G_{1}$ and $G_{2}$ be two open circular discs of radius $r$ in $D$ and let $A_{1}$ and $A_{2}$ be their centers. We shall construct a line segment in $D$ which connects $G_{1}$ and $G_{2}$. If the line segment $A_{1} A_{2}$ or some line segment parallel to it and meeting $G_{1}$ and $G_{2}$ lies in $D$, all is well. Otherwise, let $l_{t}$ denote a line segment parallel to $A_{1} A_{2}$, lying at a distance $t(0<t<r)$ on one fixed side of $A_{1} A_{2}$, and terminating in $G_{1}$ and $G_{2}$; and let $q(t)$ be the distance between $A_{1}$ and the intersection of $l_{t}$ with $S$. If $q(t)$ were a continuous function of $t, S$ would contain a nondegenerate continuum, contrary to its construction. Since $q(t)$ is bounded and discontinuous and since the set $S$ is closed, one of the lines $l_{t}$, say $l^{*}$, meets $S$ in two distinct points $P^{\prime}$ and $P^{\prime \prime}$.

Now let $P_{n}^{\prime}$ and $P_{n}^{\prime \prime}$ be the lower left-hand vertices of those squares in $S_{n}$ which contain $P^{\prime}$ and $P^{\prime \prime}$, respectively. Since $P_{n}^{\prime} \rightarrow P^{\prime}$ and $P_{n}^{\prime \prime} \rightarrow P^{\prime \prime}$ the line $\lambda_{n}$ through $P_{n}^{\prime}$ and $P_{n}^{\prime \prime}$ passes through $G_{1}$ and $G_{2}$ when $n$ is sufficiently large. Because the direction of $\lambda_{n}$ is a preferred direction, the orthogonal projection of $S$ upon a perpendicular to $\lambda_{n}$ is nowhere dense (being closed and of measure zero), and, therefore, $D$ contains a line segment parallel to $\lambda_{n}$ and meeting $G_{1}$ and $G_{2}$. This proves that $D$ is almost convex.

On the other hand, the linear projection of $S$ in the direction of a line of slope 2 is identical with the projection of the unit square $S_{0}$, 
since the projection of each of the sets $S_{n}$ is identical with it. It follows that $S$ is not of perimeter zero.

It should be remarked that every line of slope 2 which passes through the unit square meets $S$ precisely once except for a set of denumerably many lines that meet $S$ in precisely two vertices of squares of some set $S_{n}$. Also, since there are preferred directions of slope as near to 2 as we please, the set $S$ serves as an illustration of the following fact: If $m(\theta)$ is the measure of the linear projection of a bounded point-set in the direction of a line making an angle $\theta$ with the $x$-axis, then $m(\theta)$ need not be a continuous function of $\theta$.

Case (iii). Observe that the slopes of the lines through $b i$ and $b+(1-b) i$ and through $b$ and $(1-b)+i$, respectively, are $(1-2 b) / b$ and $1 /(1-2 b)$. For $n=1,2, \cdots$, the linear projection of $S_{n}$ in any direction with slope $s$, where $(1-2 b) / b \leqq s \leqq 1 /(1-2 b)$, is identical with the corresponding projection of $S_{0}$. It follows that every line of such slope that passes through the unit square between its upper left-hand and lower right-hand vertices meets $S$; hence $D$ cannot be almost convex.

\section{BIBLIOGRAPHY}

1. K. Noshiro, On the theory of schlicht functions, Journal of the Faculty of Science, Hokkaido Imperial University, Sapporo (I) vol. 2 (1934-1935) pp. 129-155.

2. S. E. Warschawski, On the higher derivatives at the boundary in conformal mapping, Trans. Amer. Math. Soc. vol. 38 (1935) pp. 310-340.

3. J. Wolff, L'intégrale d'une fonction holomorphe et d partie rélle positive dans un demi-plan est univalente, C. R. Acad. Sci. Paris vol. 198 (1934) pp. 1209-1210.

Michigan State College and UNIVERSITY OF MICHIGAN 